\title{
Variação espacial e influência do habitat na estrutura de comunidades de pequenos mamíferos em áreas de campo rupestre no Distrito Federal
}

\author{
Rodrigo Augusto Lima Santos ${ }^{1,2}$ \& Raimundo Paulo Barros Henriques ${ }^{1}$ \\ ${ }^{1}$ Departamento de Ecologia, Universidade de Brasília, \\ CP 04457, CEP 70970-919 Brasília, DF, Brasil \\ ${ }^{2}$ Autor para correspondência: Rodrigo Augusto Lima Santos, e-mail: rodrigosantos@ gmail.com
}

SANTOS, R.A.L. \& HENRIQUES, R.P.B. Spatial variation and the habitat influence in the structure of communities of small mammals in areas of rocky fields in the Federal District. Biota Neotrop. 10(1): http:// www.biotaneotropica.org.br/v10n1/en/abstract?article+bn00410012010.

\begin{abstract}
In the present study, we investigated the communities of small mammals in rocky fields in the Federal District. We examined the composition, abundance and richness and their relation to the structure of the habitat and if there is significant spatial variation in species composition among sites of rocky fields. Small mammals were sampled by live-trapping techniques in seven sites. The total effort for the seven sites was 5.680 trap-nights; we captured 157 individuals in eight species of rodents. Calomys tener and Cerradomys scotti were the most abundant species in the study sites, with 47.1 and $18.0 \%$ of total individuals, respectively. The results showed that the areas of rocky fields of the Federal District have a fauna of small mammals (average of four, range of variation of three to five species), comparable to other Cerrado physiognomies. The complexity of the habitat proved to be a major determinant in the composition of communities of small mammals in rocky fields. There was a distinction between communities of small mammals of the sites of APA Gama e Cabeça do Veado and the sites of the APA de Cafuringa. There was influence of the distance between the places on the composition of species with decreasing similarity with increasing distance among sites. The differences in environmental conditions, the structure of the habitat and the fact that it was possible two biogeographical regions may explain the faunal differences between the two regions.
\end{abstract}

Keywords: Cerrado, rodents, habitat structure, complexity.

SANTOS, R.A.L. \& HENRIQUES, R.P.B. Variação espacial e influência do habitat na estrutura de comunidades de pequenos mamíferos em áreas de campo rupestre no Distrito Federal. Biota Neotrop. 10(1): http://www.biotaneotropica.org.br/v10n1/pt/abstract?article+bn00410012010.

Resumo: No presente estudo foram investigadas as comunidades de pequenos mamíferos em campos rupestres do Distrito Federal (DF). Foram analisadas a composição, abundância e riqueza e suas relações com a estrutura do habitat e se existe variação espacial significativa na composição de espécies entre os sítios de campo rupestre. Os pequenos mamíferos foram estudados com o método de marcação e recaptura em sete sítios. O esforço total para os sete sítios foi de 5.680 armadilhas-noite, sendo capturados 157 indivíduos e oito espécies de roedores. Calomys tener e Cerradomys scotti foram as espécies mais abundantes do estudo, com 47,1 e 18,0\% do total de indivíduos, respectivamente. Ambas estavam presentes em todos os sítios amostrados. Os resultados mostraram que as áreas de campo rupestre do DF apresentam uma fauna de pequenos mamíferos (média de quatro com amplitude de variação de três a cinco espécies), comparáveis a de outras fisionomias de Cerrado. A complexidade do habitat mostrou-se como um dos principais determinantes na composição das comunidades de pequenos mamíferos em campo rupestre. Houve distinção entre as comunidades de pequenos mamíferos dos sítios da APA Gama e Cabeça de Veado e dos sítios da APA de Cafuringa. Foi constatada influência da distância entre os locais sobre a composição de espécies, com a similaridade diminuindo com o aumento da distância entre os sítios. As diferenças nas condições ambientais, na estrutura do habitat e o fato de tratar-se de duas possíveis regiões biogeográficas podem explicar as diferenças faunísticas entre as duas regiões.

Palavras-chave: Cerrado, roedores, estrutura do habitat, complexidade. 


\section{Introdução}

A diversidade e riqueza de espécies estão relacionadas principalmente, com fatores de distribuição geográficos e de estrutura do habitat (Begon et al. 2006). Em regiões sujeita à mesma história de ocupação de espécies, a estrutura do habitat é considerada como um importante determinante da riqueza e diversidade de espécies das comunidades (MacArthur et al. 1966). São variados os resultados encontrados sobre a relação entre a diversidade de espécies de diferentes grupos taxonômicos e a complexidade (variação horizontal na fisionomia) ou heterogeneidade (a variação vertical dentro do habitat) de habitat. Alguns estudos com pequenos mamíferos relatam uma relação positiva com a complexidade (Rosenzweig \& Winakur 1969), com a heterogeneidade (Fox, B.J. \& Fox, M.D. 2000) ou ambos (Williams et al. 2002).

O Cerrado possui uma rica fauna de pequenos mamíferos com várias espécies endêmicas (Marinho-Filho et al. 2002). Usando uma área de mesmo tamanho para comparação, o Cerrado apresenta uma riqueza de espécies de mamíferos comparável a da Floresta Amazônica (Mares 1992). Parte da explicação para essa alta riqueza de espécies de pequenos mamíferos no Cerrado é atribuída ao elevado grau de especificidade pelo tipo de habitat, com diferenças marcantes na composição de espécies de pequenos mamíferos entre as fisionomias vegetais (Alho et al. 1986, Mares et al. 1986, Henriques et al. 1997, Lacher Jr. \& Alho 2001). Além disso, várias espécies de pequenos mamíferos do Cerrado apresentam especialização a um determinado microhabitat, no mesmo tipo fisionômico de vegetação (Lacher Jr. \& Alho 1989, Henriques \& Alho 1991). Dessa maneira, o alto grau de especificidade das espécies de pequenos mamíferos a um determinado habitat e microhabitat associado à heterogeneidade da vegetação do Cerrado, pode explicar o aumento da riqueza, assim como a alta diversidade beta entre as comunidades do Cerrado encontrado por Marinho-Filho et al. (1994).

O bioma Cerrado apresenta uma grande variedade de formas fisionômicas variando desde formações abertas de campos até formações fechadas de floresta. Vários estudos observaram que a riqueza de pequenos mamíferos aumenta das formações de campos para as formações florestais (Alho et al. 1986, Mares et al. 1986, Henriques et al. 1997, Lacher Jr. \& Alho 2001, Vieira \& Palma 2005), sendo que a fisionomia de mata de galeria apresenta uma maior riqueza e uma composição de pequenos mamíferos diferente das outras fisionomias do Cerrado (Fonseca \& Redford 1984, Redford \& Fonseca 1986, Mares et al. 1986, Mares \& Ernest 1995, Bonvicino et al. 2005, Lacher Jr. \& Alho 2001).

As áreas de campo rupestre ocorrem acima de $900 \mathrm{~m}$ de altitude, em sua maior parte associada com a Cadeia do Espinhaço, entre $21^{\circ}$ e $10^{\circ}$ de latitude sul nos estados de Minas Gerais e Bahia e áreas disjuntas menores em Goiás (Giulietti \& Pirani 1988, Conceição et al. 2005). A vegetação de campo rupestre é formada por um mosaico de vegetação, variando de campos dominados por gramíneas até cerrado e pequenos trechos de floresta esclerofila, determinada por diferenças na topografia, inclinação do terreno, substrato e micro-clima (Harley 1988). Essas áreas estão associadas com uma das mais altas porcentagens de endemismo da flora brasileira (Giulietti \& Pirani 1988, Conceição et al. 2005).

Existem poucos estudos sobre a fauna de pequenos mamíferos nos campos rupestres. Os estudos anteriores mostram que os campos rupestres apresentam uma riqueza de pequenos mamíferos comparável com áreas de cerrado, com quatro a seis espécies de roedores e duas a quatro espécies de marsupiais (Dietz 1983, Oliveira \& Pessôa 2005, Bonvicino et al. 2002). Foi sugerido que as áreas de campos rupestres em maiores altitudes podem conter uma fauna de pequenos mamíferos endêmicos não existentes nas áreas de cerrado em baixa altitude (Bonvicino et al. 2005). Apesar do pequeno número de inventários foi indicada uma espécie de roedor (Oligoryzomys rupestris) como endêmica para esse tipo de vegetação (Bonvicino et al. 2002, 2005, Weksler \& Bonvicino 2005).

Ao comparar comunidades de pequenos mamíferos no Cerrado pode-se notar que algumas espécies são abundantes em determinados habitats e ausentes em outros, como por exemplo, o roedor Thrichomys apereoides particularmente abundante em ambientes rochosos (Lacher Jr. \& Alho 1989). Dessa maneira, as características estruturais do ambiente são um dos fatores que podem influenciar na composição, distribuição e na abundância de pequenos mamíferos. O objetivo do presente estudo foi caracterizar as comunidades de pequenos mamíferos em sete sítios de campo rupestre, em duas áreas do Distrito Federal, avaliando a hipótese de que existe relação entre a estrutura do habitat e a composição de espécies. Especificamente foram analisadas: 1) A composição e abundância das espécies; 2) as características dos habitats influenciam a composição de espécies nos campos rupestres; e 3) se existe variação espacial significativa na composição de espécies entre os sítios de campo rupestre.

\section{Material e Métodos}

\section{1. Área de estudo}

Esse estudo foi realizado em duas áreas do Distrito Federal (DF): a Área de Proteção Ambiental (APA) Gama e Cabeça de Veado, localizada na região centro sul e a APA de Cafuringa, localizada na região noroeste (Figura 1). Foram realizados levantamentos em três sítios na APA Gama e Cabeça de Veado: dois sítios na Fazenda Água Limpa da Universidade de Brasília (FAL 1 e FAL 2) e um no Jardim Botânico de Brasília (JBB). Na APA de Cafuringa foram realizados levantamentos em quatro sítios: dois sítios na fazenda Chapada Imperial (CI 1 e CI 2) e dois na área de Mumunhas (MU 1 e MU 2). O clima da APA Gama e Cabeça de Veado apresenta a precipitação média anual de $1.453 \mathrm{~mm}$ e temperatura média anual é de $22.1^{\circ} \mathrm{C}$ (Dados obtidos na estação meteorológica da Reserva Ecológica do IBGE). O clima da APA de Cafuringa apresenta precipitação média anual superior à registrada para a APA Gama e Cabeça de Veado, com valores entre 1.650 a $1.700 \mathrm{~mm}$ e temperatura média de $20^{\circ} \mathrm{C}$ (Fortes et al. 2007).

\section{Programa de captura}

Os levantamentos de pequenos mamíferos foram realizados durante os meses de maio de 2008 a fevereiro de 2009. Em cada sítio foram estabelecidas duas linhas de $90 \mathrm{~m}$ de comprimento, afastadas $30 \mathrm{~m}$ uma da outra. Cada linha era composta de 10 estações de captura, distantes $10 \mathrm{~m}$ entre si, com um total de 20 estações de captura por sítio. Em cada estação de captura foram colocadas duas armadilhas Sherman $(23 \times 9 \times 8 \mathrm{~cm})$, ambas no solo, totalizando 40 armadilhas por sítio. As armadilhas foram iscadas com uma mistura de sardinha em lata, creme de amendoim e fubá, sendo vistoriadas no início da manhã de cada dia de captura. Foram realizados quatro períodos de capturas em cada sítio durante o estudo. O esforço de captura variou de 760 a 920 armadilhas-noite por sítio, com um esforço total de 5.680 armadilhas/noite no período do estudo. Os animais capturados foram identificados e marcados na orelha com uma etiqueta metálica numerada (National Band \& Tags - Mod. 1005 - 1). Após a coleta dos dados os animais foram soltos nos locais onde foram capturados. Foram coletados espécimes testemunhos, as quais foram depositadas na coleção de Zoologia da Universidade de Brasília. 


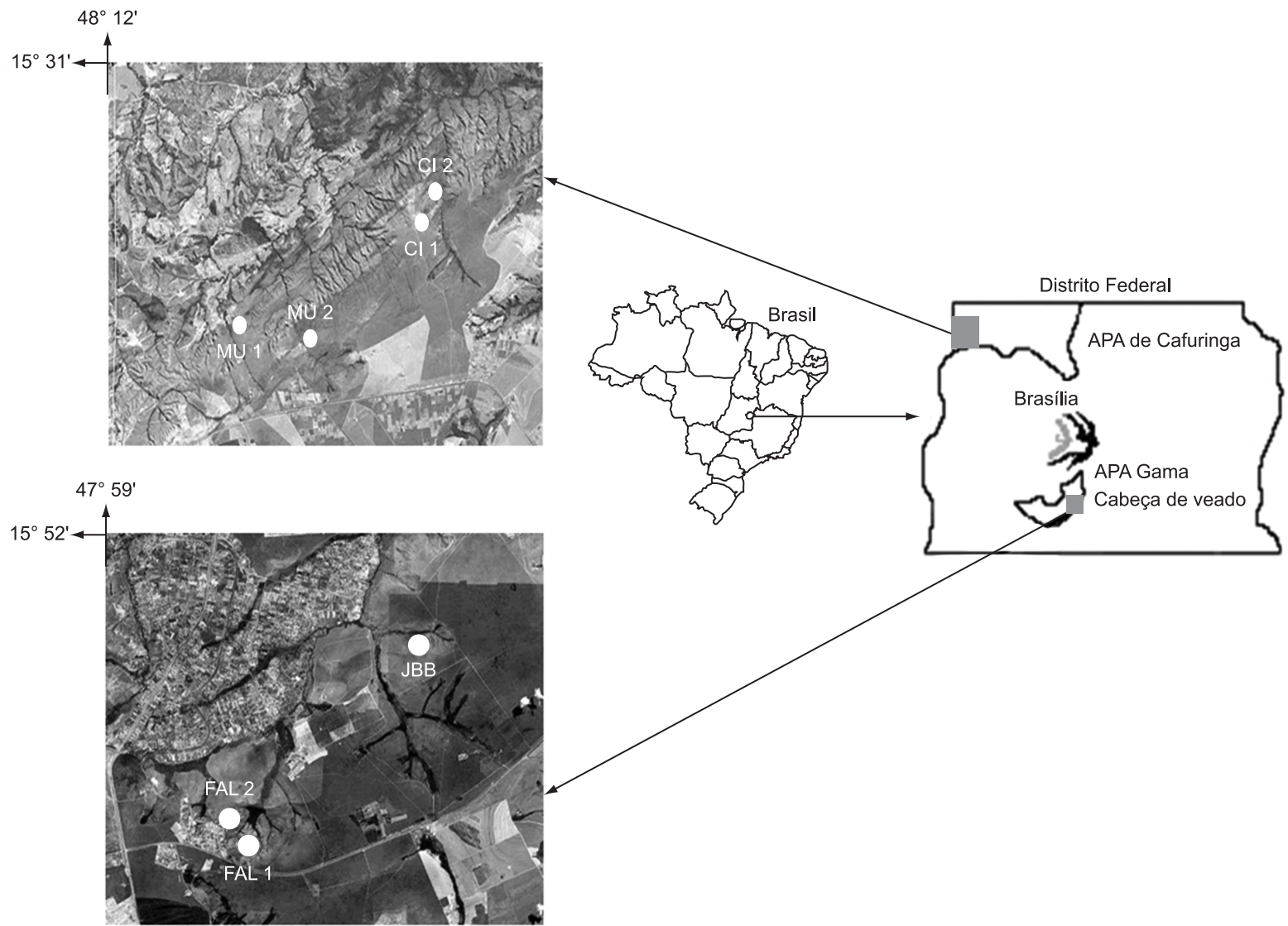

Figura 1. Localização geográfica da área de estudo mostrando os sítios da APA de Cafuringa (CI 1, CI 2, MU 1 e MU 2) e os sítios da APA Gama e Cabeça de Veado (FAL 1, FAL 2 e JBB). Código das siglas da área de estudo: FAL (Fazenda Água Limpa - UnB), JBB (Jardim Botânico de Brasília), CI (Chapada Imperial), MU (Mumunhas).

Figure 1. Location of the study area showing the sites of the APA de Cafuringa (CI 1, CI 2, MU 1 and MU 2) and the sites of APA Gama e Cabeça de Veado (FAL 1, FAL 22 and JBB). Code of acronyms of the study area: FAL (Fazenda Água Limpa - UnB), JBB (Jardim Botânico de Brasília), CI (Chapada Imperial), MU (Mumunhas).

\section{Análise dos dados}

A riqueza de espécies foi estimada através de índices extrapoladores não paramétricos, Jackknife (I e II), Chao (I e II) e Bootstrap (Magurran 2003, Colwell 2005). Tais estimadores permitem comparar dados obtidos com métodos e esforço de coleta diferente, e sua vantagem é o cálculo dos limites de confiança da estimativa. Para a determinação dos índices e intervalo de confiança foram realizadas 100 simulações utilizando o programa EstimateS versão 8.0 (Colwell 2005).

A riqueza de espécies para cada sítio também foi avaliada usando o método de rarefação (Sanders 1968). O método de rarefação estima a riqueza esperada de espécies para um subconjunto de indivíduos amostrados aleatoriamente do total de uma comunidade. Foi usado o programa EcoSim ${ }^{\circledR}$ (Gotelli \& Entsminger 2004), para amostrar por repetição (1.000 vezes) um valor crescente até o número total de indivíduos por sítio.

A estrutura do habitat foi descrita através de levantamentos realizados no campo. Em cada sítio foram estabelecidas quatro transeções de $50 \times 2 \mathrm{~m}\left(100 \mathrm{~m}^{2}\right)$, totalizando uma área de $400 \mathrm{~m}^{2}$ por sítio. Em cada transeção para cada planta foi medida a circunferência ao nível do solo para caules com mais de $15 \mathrm{~cm}$ de circunferência, a altura das plantas e a extensão da copa que interceptava a transeção. Além disso, foi medida a extensão do solo que era coberta com rocha na transeção. Com esses dados foram calculadas para cada sítio as seguintes variáveis:

1. Área Basal média ao nível do solo $\left(\mathrm{ABA}, \mathrm{m}^{2}\right)$;

2. Altura média das plantas arbustivo-arbóreas (ALT, m);
3. Número médio de plantas arbustivo-arbóreas (NPL);

4. Porcentagem da cobertura da copa das plantas arbustivoarbóreas (COPA, \%), usando o método de interceptação em linha (Müller-Dombois \& Ellemberg 1974), calculadas para quatro linhas de $50 \mathrm{~m}$ de comprimento; e

5. Porcentagem da cobertura de rocha (ROCHA, \%), usando o método de interceptação em linha (Müller-Dombois e Ellemberg 1974), calculados para quatro linhas de $50 \mathrm{~m} \mathrm{de}$ comprimento.

Os valores médios dos descritores ambientais de cada sítio encontram-se na Tabela 1.

Para reduzir o conjunto de variáveis ambientais foi realizada uma analise de componentes principais (PCA). A matriz de dados ambientais incluiu cobertura de rocha (ROCHA), cobertura de copa (COPA), altura (ALT), área basal (ABA) e número de plantas (NPL). Os dados de porcentagem de cobertura de rocha e de copa foram transformados em arco seno $(\sqrt{ } p)$, uma vez que dados em \% não são indicados para análises estatísticas (Zar 1999). O primeiro eixo da PCA foi considerado como a variação ambiental mais expressiva do complexo gradiente ambiental dos sítios.

Foram realizadas duas analises multivariadas, analise de agrupamento e analise de escalonamento multidimensional (MDS), para ordenar e agrupar as comunidades de pequenos mamíferos. Para as análises foi preparada uma matriz de presença e ausência das espécies nos sítios. 
Tabela 1. Média e desvio padrão das variáveis ambientais dos sete sítios.

Table 1. Mean and standard deviation of environmental variables of the seven sites.

\begin{tabular}{lccccc}
\hline Local & Área Basal $\left(\mathbf{m}^{2}\right)$ & Altura $(\mathbf{m})$ & Copa $(\%)$ & Rocha $(\%)$ & Número de plantas \\
\hline FAL I & $0,37 \pm 0,15$ & $0,8 \pm 0,27$ & $31,8 \pm 9,6$ & $15,7 \pm 17,3$ & $94,7 \pm 32,4$ \\
FAL II & $0,38 \pm 0,12$ & $0,9 \pm 0,23$ & $32,5 \pm 14,3$ & $23,3 \pm 14,3$ & $72,8 \pm 14,2$ \\
JBB & $0,23 \pm 0,03$ & $1,2 \pm 0,47$ & $29,5 \pm 15,8$ & $30,7 \pm 16,35$ & $35,7 \pm 14,6$ \\
MU 1 & $0,31 \pm 0,11$ & $2,1 \pm 0,11$ & $48,5 \pm 18,3$ & $2,6 \pm 2,17$ & $37,5 \pm 7,8$ \\
MU 2 & $0,15 \pm 0,04$ & $1,8 \pm 0,25$ & $30,9 \pm 16,4$ & $7,8 \pm 2,13$ & $36,7 \pm 9,8$ \\
CI 1 & $0,11 \pm 0,04$ & $1,6 \pm 0,16$ & $24,3 \pm 4,1$ & $2,49 \pm 4,3$ & $28,7 \pm 16,7$ \\
CI 2 & $0,13 \pm 0,06$ & $1,3 \pm 0,10$ & $23,0 \pm 8,3$ & $2,2 \pm 1,5$ & $33,2 \pm 12$ \\
\hline
\end{tabular}

$\mathrm{Na}$ análise de agrupamento o algoritmo de agrupamento empregado foi o da Média Ponderada (UPGMA) (Sneath e Sokal 1973), com o índice de similaridade de Sørensen. Foram realizadas duas Análises de Agrupamento: 1) Uma agrupando sítios a partir da presença das espécies de pequenos mamíferos; e 2) outra agrupando as espécies em função de sua ocorrência nos sítios. Em cada uma das análises, a fim de classificar as unidades de análises em grupos, calculou-se a média dos valores de similaridade para cada reunião de unidades.

Para a analise de ordenação MDS foi utilizado à distância de Jaccard para definir possíveis padrões de organização, tanto para os sítios como para as espécies. A análise do escalonamento multidimensional foi utilizada como uma técnica complementar à análise de agrupamento para encontrar uma configuração de pontos que permitam visualizar o comportamento dos sítios e das espécies em um número menor de dimensões. Para poder mensurar a qualidade do ajuste foi utilizado à medida stress, que indica a proporção da variação das distâncias originais em relação às distâncias preditas pelo MDS.

A quantificação da influência das características ambientais (ABA, ALT, COPA, ROCHA e NPL) na composição das comunidades de pequenos mamíferos foi analisada através de uma regressão linear entre os valores obtidos no eixo único do MDS para os sítios com o primeiro eixo da PCA das variáveis ambientais.

A influência da autocorrelação espacial sobre a composição de espécies foi analisada através de um teste de Mantel quantificando a influência da distância entre os locais amostrados sobre a similaridade na composição de espécies entre os sítios de campo rupestre.

Para todas as análises, foi utilizado o nível de significância de $5 \%$. Os cálculos estatísticos foram realizados usando o programa PC-ORD versão 4.0 (McCune \& Mefford 1999).

\section{Resultados}

Foi registrado um total de 157 indivíduos com 320 capturas (Tabela 2). O sucesso de captura por sítio variou de 1,3\% em MU 2 e $4,8 \%$ na FAL 1, resultando em um sucesso total de capturas de $2,8 \%$. A FAL 1 foi o sítio que apresentou maior número de indivíduos (39) e de número de capturas (82) respectivamente. MU 2 foi o sítio que apresentou o menor número de indivíduos (10) e de capturas (16).

Oito espécies de pequenos mamíferos (Tabela 2) foram registradas, todas roedores, das quais duas ocorreram em todos os sítios (Calomys tener e Cerradomys scotti). Algumas espécies foram de ocorrência restrita a APA Gama e Cabeça de Veado como Thalpomys lasiotis, Oligoryzomys fornesi e Calomys expulsus que foi capturado apenas na FAL 2. Calomys tocantinsi e Thrichomys apereoides foram registrados somente na APA de Cafuringa, com a segunda ocorrendo apenas em MU 2. Calomys tener foi à espécie mais abundante com 74 indivíduos $(47,1 \%)$, seguida por Cerradomys scotti $(18,4 \%)$, Necromys lasiurus (16,5\%), Oligoryzomys fornesi (5,7\%), Calomys

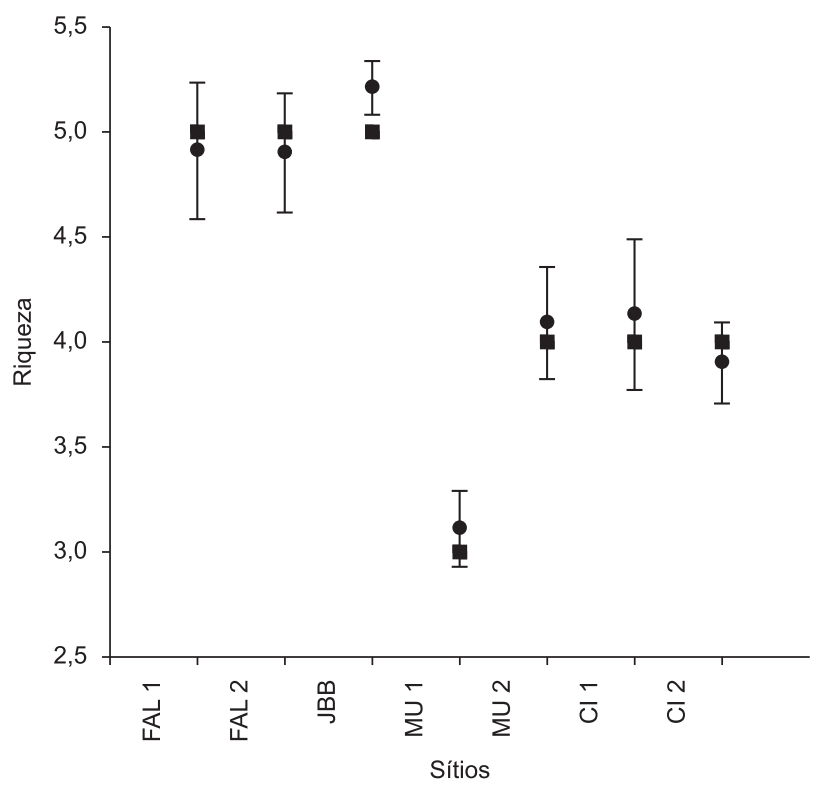

Figura 2. Riqueza de espécies observada e estimada nos sete sítios do estudo. As barras indicam a riqueza média e desvio padrão de todos os estimadores e o quadrado indica a riqueza observada nos sítios.

Figure 2. Richness of species observed and estimated in the seven study sites. Bars indicate the wealth means and standard deviations of all estimators and the square indicates the richness observed in sites.

tocantinsi (5\%), Thalpomys lasiotis (3,8\%), Thrichomys apereoides $(2,5 \%)$, Calomys expulsus $(0,6 \%)$.

A riqueza de espécies observada variou de três a cinco por sítio. Os sítios localizados na APA Gama e Cabeça de Veado apresentaram maior riqueza, com cinco espécies em cada sitio (Figura 2). Os sítios da APA de Cafuringa apresentaram menor riqueza variando de três a quatro, sendo que MU 2 foi o local que registrou a menor riqueza, com apenas três espécies.

As estimativas de riqueza obtidas pelos diferentes índices mostraram que o número observado de espécies correspondeu de 86,3 a $100 \%$ (média 99,02 \pm 1,5\%) das espécies estimadas para cada sítio (Figura 2). Esses resultados indicam que a maior parte das espécies que ocorreram nos sítios foi capturada nos levantamentos. O sítio do JBB apresentou a maior riqueza de espécies estimada (intervalo esperado de riqueza de 5,06 - 5,41 espécies) calculados com os estimadores Jackknife I, Chao I, Chao II e Bootstrap, com exceção do estimador Jackknife II para o sítio FAL 2 (5,35 espécies). O sítio que apresentou a menor riqueza de espécies usando todos os estimadores foi MU 1 (2,90 - 3,28 espécies).

As curvas de rarefação de espécies de pequenos mamíferos para cada sítio são mostradas na Figura 3. Quando comparamos as curvas de rarefação da riqueza de espécies para cada sítio com valor 
Tabela 2. Número de indivíduos $(\mathrm{N})$ e número de capturas $(\mathrm{C})$ de pequenos mamíferos para sete sítios de campo rupestre no Distrito Federal.

Table 2. Number of individuals $(\mathrm{N})$ and number of captures $(\mathrm{C})$ of small mammals in seven rocky field sites in Federal District.

\begin{tabular}{|c|c|c|c|c|c|c|c|c|c|c|c|c|c|c|c|c|}
\hline \multirow[t]{2}{*}{ Espécie } & \multicolumn{2}{|c|}{ FAL 1} & \multicolumn{2}{|c|}{ FAL 2} & \multicolumn{2}{|c|}{ JBB } & \multicolumn{2}{|c|}{ MU 1} & \multicolumn{2}{|c|}{ MU 2} & \multicolumn{2}{|c|}{ CI 1} & \multicolumn{2}{|c|}{ CI 2} & \multicolumn{2}{|c|}{ Total } \\
\hline & $\mathbf{N}$ & $\mathbf{C}$ & $\mathbf{N}$ & $\mathrm{C}$ & $\mathbf{N}$ & $\mathbf{C}$ & $\mathbf{N}$ & C & $\mathbf{N}$ & $\mathbf{C}$ & $\mathbf{N}$ & C & $\mathbf{N}$ & C & $\mathbf{N}$ & C \\
\hline $\begin{array}{l}\text { Calomys tener } \\
\text { (Winge, 1887) }\end{array}$ & 29 & 70 & 17 & 21 & 4 & 7 & 10 & 21 & 1 & 1 & 5 & 10 & 8 & 11 & 72 & 141 \\
\hline $\begin{array}{l}\text { Cerradomys scotti } \\
\text { (Langguth e Bonvicino, 2003) }\end{array}$ & 4 & 7 & 8 & 25 & 2 & 6 & 4 & 9 & 3 & 6 & 5 & 7 & 3 & 3 & 29 & 63 \\
\hline $\begin{array}{l}\text { Necromys lasiurus } \\
\text { (Lund, 1841) }\end{array}$ & 1 & 1 & 2 & 4 & 7 & 32 & - & - & 2 & 5 & 6 & 14 & 8 & 14 & 26 & 70 \\
\hline $\begin{array}{l}\text { Calomys expulsus } \\
\text { (Lund, 1841) }\end{array}$ & - & - & 1 & 1 & - & - & - & - & - & - & - & - & - & - & 1 & 1 \\
\hline $\begin{array}{l}\text { Calomys tocantinsi } \\
\text { (Bonvicino et al., 2003) }\end{array}$ & - & - & - & - & - & - & 3 & 3 & - & - & 2 & 2 & 3 & 4 & 9 & 9 \\
\hline $\begin{array}{l}\text { Oligoryzomys fornesi } \\
\text { (Massoia, 1973) }\end{array}$ & 3 & 3 & 4 & 4 & 2 & 2 & - & - & - & - & - & - & - & - & 9 & 9 \\
\hline $\begin{array}{l}\text { Thalpomys lasiotis } \\
\text { (Thomas, 1916) }\end{array}$ & 2 & 2 & - & - & 4 & 21 & - & - & - & - & - & - & - & - & 6 & 23 \\
\hline $\begin{array}{l}\text { Thrichomys apereoides } \\
\text { (Lund, 1841) }\end{array}$ & - & - & - & - & - & - & - & - & 4 & 4 & - & - & - & - & 4 & 4 \\
\hline Total & 39 & 83 & 32 & 55 & 19 & 68 & 17 & 33 & 10 & 16 & 18 & 33 & 22 & 32 & 157 & 320 \\
\hline Esforço de captura & \multicolumn{2}{|c|}{800} & \multicolumn{2}{|c|}{920} & \multicolumn{2}{|c|}{920} & \multicolumn{2}{|c|}{760} & \multicolumn{2}{|c|}{760} & \multicolumn{2}{|c|}{760} & \multicolumn{2}{|c|}{760} & \multicolumn{2}{|c|}{5680} \\
\hline Sucesso de captura (\%) & \multicolumn{2}{|c|}{4,75} & \multicolumn{2}{|c|}{3,37} & \multicolumn{2}{|c|}{1,74} & \multicolumn{2}{|c|}{2,23} & \multicolumn{2}{|c|}{1,31} & \multicolumn{2}{|c|}{2,36} & \multicolumn{2}{|c|}{2,89} & \multicolumn{2}{|c|}{2,8} \\
\hline Altitude (m) & \multicolumn{2}{|c|}{1200} & \multicolumn{2}{|c|}{1203} & \multicolumn{2}{|c|}{1100} & \multicolumn{2}{|c|}{1065} & \multicolumn{2}{|c|}{1231} & \multicolumn{2}{|c|}{1160} & \multicolumn{2}{|c|}{1202} & \multicolumn{2}{|c|}{ - } \\
\hline Coordenadas geográficas & $15^{\circ} 5$ & $43 " s$ & $15^{\circ} 5$ & $98 " \mathrm{~s}$ & $15^{\circ} 5$ & $24 " \mathrm{~S}$ & $15^{\circ} 3$ & $73 " \mathrm{~s}$ & $15^{\circ} 3$ & $64 " \mathrm{~S}$ & $15^{\circ} 3$ & $91 " \mathrm{~S}$ & $15^{\circ}$ & $27 " \mathrm{~s}$ & - & 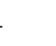 \\
\hline & $47^{\circ} 5$ & $38 ” O$ & $47^{\circ} 5$ & 39 " O & $47^{\circ} 5$ & $47 ” O$ & $48^{\circ} 1$ & $69 " \mathrm{O}$ & $48^{\circ} 0$ & $31 " \mathrm{O}$ & $48^{\circ} 0$ & $49 " \mathrm{O}$ & $48^{\circ} \mathrm{C}$ & $40 " \mathrm{O}$ & - & - \\
\hline
\end{tabular}

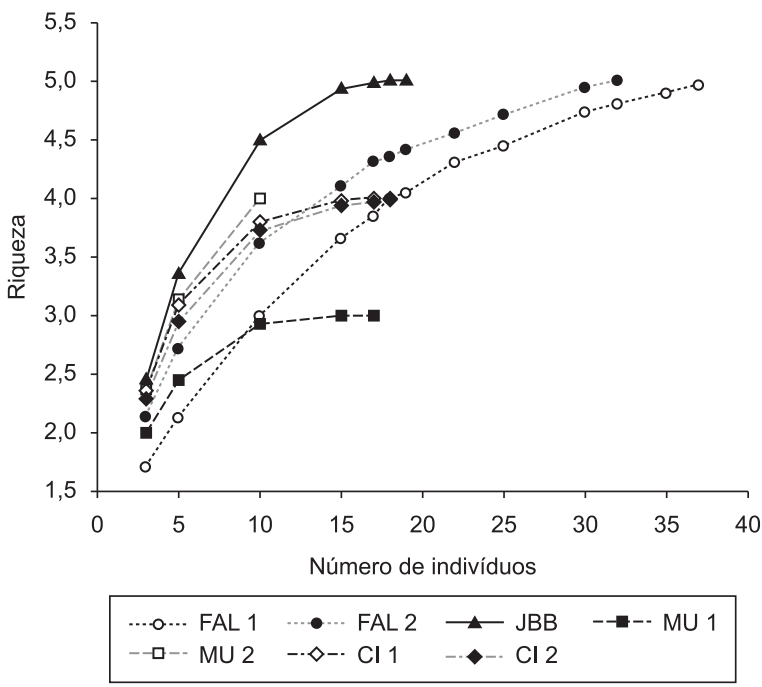

Figura 3. Curvas de rarefação de espécies de pequenos mamíferos baseada no número de indivíduos em sete sítios de campo rupestre no Distrito Federal.

Figure 3. Rarefaction curves of species of small mammals based on the number of individuals in seven sites of rocky fields in Distrito Federal.

crescente até um total de 10 indivíduos, o JBB apresentou a maior riqueza, sendo que FAL 1 e MU 1 apresentaram a menor riqueza. Usando maior número de indivíduos, até 20 indivíduos, o JBB teve a maior riqueza de espécies e MU 1 a menor riqueza de espécies. Esses resultados são consistentes com os obtidos usando os estimadores não paramétricos.

A análise de agregação separou os sítios em dois grupos distintos: (1) A APA de Cafuringa ao norte do Distrito Federal (MU 1, CI 1, CI 2) e (2) da APA Gama e Cabeça do Veado no centro sul do Distrito Federal (Figura 4a). No entanto, o sítio de MU 2, embora tenha uma similaridade maior com os sítios da APA do Cafuringa,
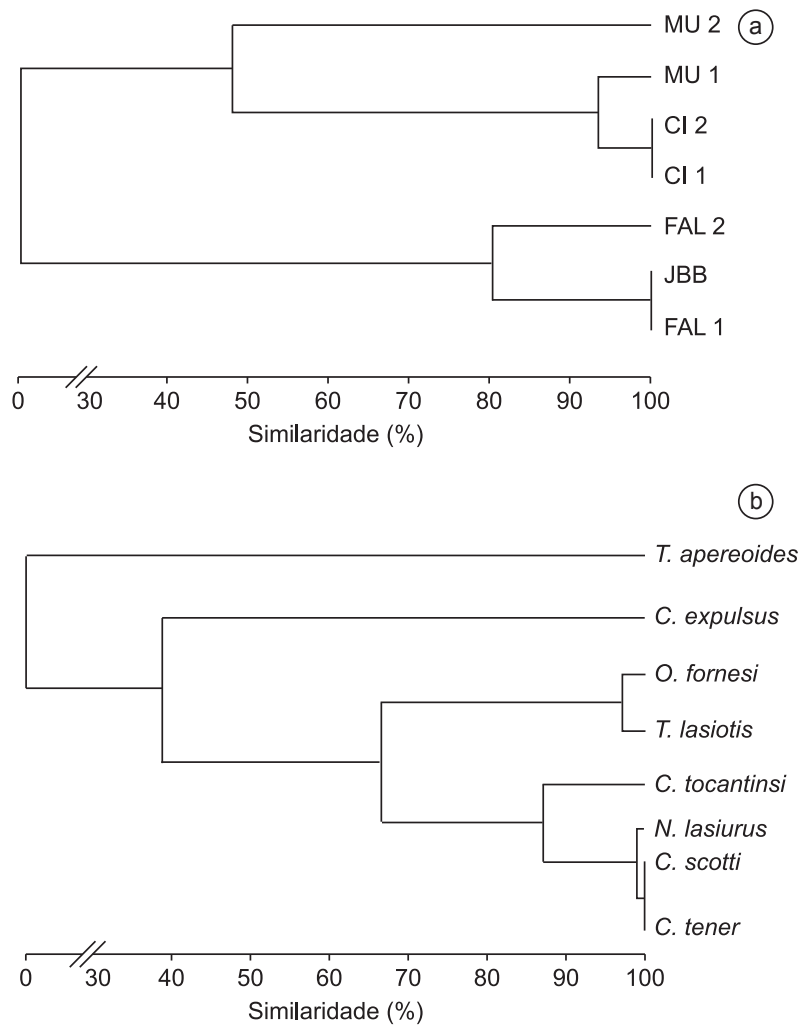

Figura 4. Dendograma de similaridade obtido através do coeficiente de Sørensen e UPGMA: (a) similaridade entre os sítios de estudo; (b) relações entre as espécies de roedores. A linha tracejada indica o ponto de corte para formação dos grupos.

Figure 4. Dendrogram of similarity obtained by the coefficient of Sørensen and UPGMA: (a) Similarity among the sites of study, (b) relationships between species of rodents. The dashed line indicates the cutoff point for formation of groups. 
Santos, R.A.L. \& Henriques, R.P.B.
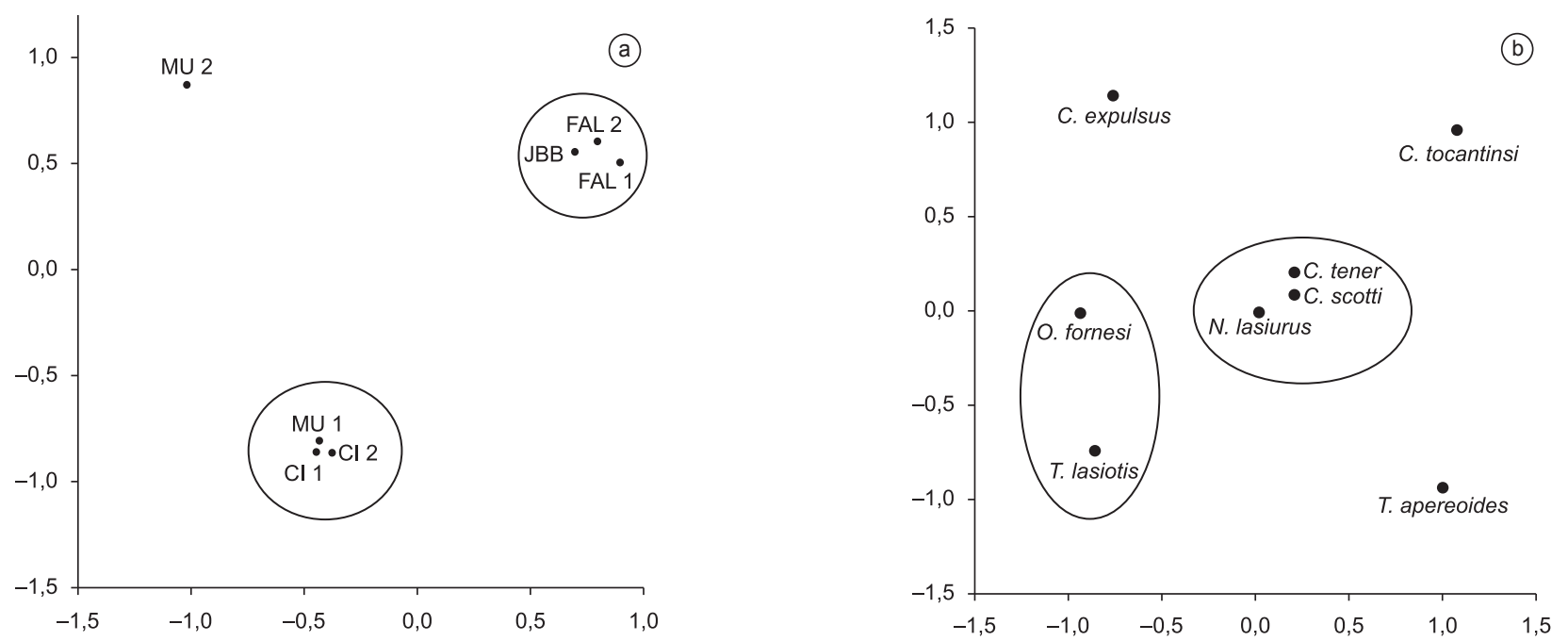

Figura 5. Ordenação resultante da analise de escalonamento multidimensional (MDS) dos sítios (a) e das espécies (b). Os círculos indicam os grupos formados a partir do agrupamento. Os ambientes com maior cobertura de rocha e mais próximos geograficamente (FAL 1, FAL 2 e JBB) são representados mais à cima do eixo Y no gráfico a, enquanto que as espécies mais abundantes do estudo são representadas mais à direita do eixo $\mathrm{X}$ no gráfico b.

Figure 5. Ordination resulting from the analysis of multidimensional scaling (MDS) of sites (a) and species (b). The circles indicate the groups formed from the pool. Environments with greater coverage of rock and geographically closer (FAL 1, FAL 2 and JBB) are represented over the top of the Y axis in the chart, while generalist species and most abundant of the study are represented to the right of the $\mathrm{X}$ the graph $\mathrm{b}$.

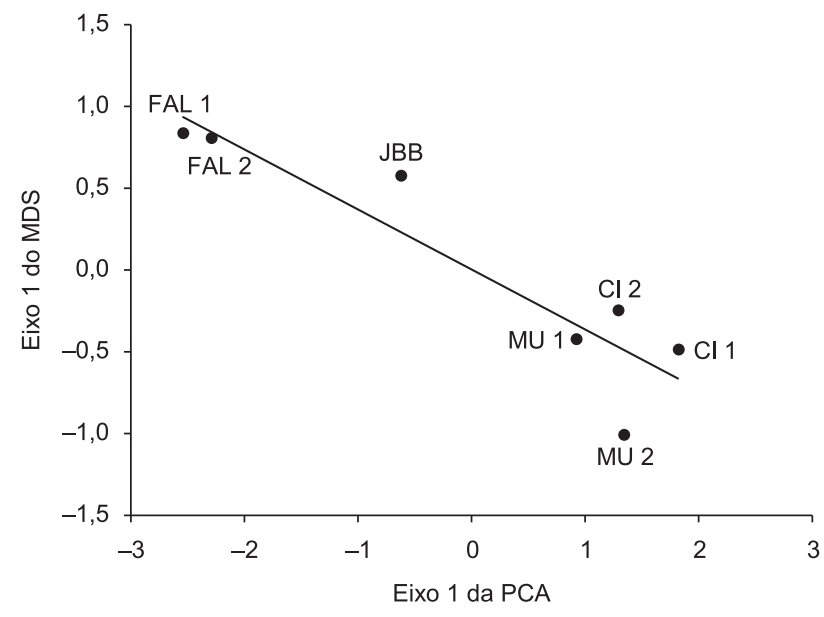

Figura 6. Primeiro eixo do MDS dos sítios versus gradiente de complexidade ambiental definido pelo primeiro eixo da PCA $(r=0,91, \mathrm{p}=0,002)$. O primeiro eixo do MDS representa a composição da estrutura das comunidades. O primeiro eixo da PCA representa um gradiente de complexidade com aumento da altura das plantas da esquerda para direita e aumento do número de plantas, área basal e cobertura de rocha da direita para esquerda.

Figure 6. The first axis of the MDS of sites versus complex environmental gradient defined by the first axis of PCA $(r=0.91, p=0.002)$. The first MDS represents the composition of the communities. The first PCA axis represents a gradient with increased plant height from left to right and increase the number of plants, basal area and cover rock from right to left.

apresentou o valor de similaridade abaixo do valor médio de corte para os grupos, indicando uma maior individualidade da fauna de pequenos mamíferos. No caso dos sítios da CI 1 e CI 2 a similaridade da fauna de pequenos mamíferos esteve relacionada com a proximidade geográfica e em outro caso não esteve relacionada, como entre FAL 1 que teve maior similaridade com a fauna do JBB do que com FAL 2 mais próximo.

As espécies de pequenos mamíferos foram separadas em dois grupos (Figura 4b): o (1) formado por Calomys tener, Cerradomys scotti, Calomys tocantinsi e Necromys lasirus, e (2) por Oligoryzomys fornesi e Thalpomys lasiotis. As espécies Thrichomys apereoides e Calomys expulsus, não foram agregadas em nenhum grupo.

A análise de escalonamento multidimensional MDS resultou em uma ordenação das espécies e sítios em duas dimensões, com stress de 0,15 e 0,22 , respectivamente (Figura 5). A ordenação produzida para as espécies consegue distinguir satisfatoriamente dois grupos definidos e para a ordenação dos sítios também é possível visualizar dois grupos distintos. Os resultados obtidos foram similares com os grupos definidos pela analise de agrupamento.

O primeiro eixo da PCA, representando a complexidade da vegetação, explicou 56,35\% da variância nos dados (eigenvalue 2,818). O primeiro eixo da ordenação foi correlacionado negativamente com o número de plantas $(-0,539)$, área basal $(-0,516)$, cobertura de rocha $(-0,437)$ e cobertura de copa $(-0,072)$ e positivamente com a altura das plantas $(0,496)$.

A composição da estrutura das comunidades definida pelo primeiro eixo do MDS variou negativamente com o gradiente de complexidade ambiental definido pelo primeiro eixo da PCA (Figura 6). Esta relação foi linear e estatisticamente significativa $(\mathrm{r}=0,91, \mathrm{p}=0,002)$.

Foi constatada influência da distância entre os locais sobre a composição de espécies (Mantel $\mathrm{r}=-0,68 ; \mathrm{P}=0,02$ ), ou seja, sítios mais próximos são mais similares na composição de espécies conforme observado no dendrograma de similaridade com a diferenciação entre as duas regiões (APA de Cafuringa e APA Gama e Cabeça de Veado).

\section{Discussão}

Os números de espécies registradas para os sítios de estudo no Distrito Federal variaram de três a cinco espécies por sítio e oito espécies no total. Números semelhantes de riqueza variando de seis a dez espécies foram registrados para as áreas de campo rupestre em outros estudos por Dietz (1983) e Bonvicino et al. (2002, 2005). Embora, as poucas informações não permitam fazer generalizações, a riqueza de espécies é comparável à registrada para comunidades de pequenos mamíferos para áreas de cerrado sensu stricto (Mares et al. 1986, Henriques et al. 1997, 2000, Vieira \& Marinho-Filho 
1998, Vieira 1999, Jorge et al. 2001, Lacher Jr. \& Alho 2001, Ribeiro \& Marinho-Filho 2005).

Das oito espécies de pequenos mamíferos registradas nos sete sítios de campo rupestre no Distrito Federal, sete já haviam sido registradas anteriormente em outras áreas de campo rupestre (Dietz 1983, Oliveira \& Pessôa 2005, Bonvicino et al. 2002, 2005, Weksler \& Bonvicino 2005). As espécies mais abundantes registradas nesse estudo foram: Calomys tener e Cerradomys scotti, que ocorreram em todos os sítios. Outras espécies tiveram uma ocorrência restrita a uma das APAs como Calomys tocantinsi e Thrichomys apereoides, que foi capturado em apenas um sítio (MU 2) na APA de Cafuringa. Thalpomys lasiotis e Oligoryzomys fornesi foram capturados apenas na APA do Gama e Cabeça do Veado. Os registros anteriores de Calomys tocantinsi ocorreram no cerrado da região do Formoso do Araguaia - TO e em Mato Grosso (Bonvicino et al. 2003) tanto em formações abertas como florestais.

Em ambientes com alta cobertura de afloramentos rochosos, o roedor Thrichomys apereoides é particularmente freqüente, em relação ao campo sujo, cerrado sensu stricto e cerradão (Lacher Jr. \& Alho 2001, Bonvicino et al. 2002). No presente estudo foram registrados poucos indivíduos da espécie, apenas em um sítio, MU 2. Thrichomys apereoides foi registrado no Distrito Federal na década de 80, nas áreas da APA Gama e Cabeça de Veado (Fonseca \& Redford 1984, Mares et al. 1986, Alho et al. 1986) e recentemente na Estação Ecológica Águas Emendadas (ESECAE) próxima a APA de Cafuringa (Marinho-Filho et al. 1998).

A espécie mais abundante neste estudo, Calomys tener, têm ampla ocorrência no Cerrado, sendo registrada desde áreas abertas de campo sujo até cerradão, com maior ocorrência em áreas de cerrado sensu stricto (Alho 1981, Mares et al. 1986, Alho et al. 1986, Henriques et al 1997, 2000). A outra espécie de roedor mais abundante neste estudo, Cerradomys scotti, foi observada desde áreas de campo limpo até cerradão (Henriques et al. 1997, Lacher Jr. \& Alho 2001, Briani et al. 2004).

Em um estudo das comunidades de mamíferos nos llaños da Venezuela, August (1983) encontrou uma correlação positiva da riqueza total de mamíferos com a complexidade do habitat, mas não observou este padrão quando aplicado apenas para os pequenos mamíferos. Já Kerley (1992) em um estudo conduzido no semi-árido sul africano observou que a diversidade de pequenos mamíferos aumentou com a complexidade do habitat, expresso em termos de diversidade de altura foliar (DAF) de Mac Arthur, R.H. \& Mac Arthur, J.W. (1961), e também com o aumento da cobertura de rocha. Em outro estudo realizado no deserto da Califórnia a complexidade do habitat medida pelo número de espécies de plantas e o DAF do habitat, foi menos importante para explicar a diversidade de pequenos mamíferos do que a previsibilidade na precipitação, estimada pela diferença entre a média e o desvio padrão da precipitação anual (Brown 1973). Os resultados obtidos mostraram que a complexidade do habitat é um dos principais determinantes na composição das comunidades de pequenos mamíferos de campo rupestre, corroborando com a hipótese proposta.

Tanto a análise de agrupamento para os sítios (Figura 4a) como a ordenação gerada pelo MDS (Figura 5) revelou a existência de dois agrupamentos de sítios: (1) APA de Cafuringa e (2) APA Gama e Cabeça de Veado. Essas duas regiões também apresentam diferenças nas condições climáticas, com a APA de Cafuringa localizada a noroeste do Distrito Federal apresentando uma média pluviométrica maior do que a APA Gama e Cabeça de Veado. Vários estudos comparando as áreas ao norte com as áreas centro sul do Distrito Federal indicaram uma diferenciação na composição da fauna e flora. Para fauna de lagartos (Colli 2005) e de borboletas (Brown \& Gifford 2002) foi observada uma diferença na composição de espécies entre as duas regiões. No estudo de Ratter et al. (2003), a região a noroeste do Distrito Federal abrangendo a área da APA de Cafuringa mostrou diferenças na composição de espécies de plantas lenhosas a regiões ao centro sul do DF. Os resultados deste estudo indicam que a diferença na composição de pequenos mamíferos encontradas resulta tanto da diferença na estrutura do habitat como da distância entre as áreas $(\sim 50 \mathrm{Km})$ com a APA de Cafuringa pertencete à bacia de drenagem que se dirige ao norte em direção a Bacia do Rio Tocantins e a APA Gama e Cabeça de Veado pertencente a bacia de drenagem do Rio São Bartolomeu que segue em direção a bacia do Rio Paraná ao Sul. Os dados da literatura e os resultados obtidos sugerem que a APA de Cafuringa e a APA Gama e Cabeça de Veado pertencem a duas regiões biogeográficas distintas.

Alguns trabalhos mostram que as espécies de pequenos mamíferos do Cerrado podem ser separadas em dois grupos de espécies: um formado por espécies que ocorrem apenas no cerrado sensu lato e outro grupo formado por espécies restritas as matas de galeria (Mares et al. 1986, Lacher Jr. \& Alho 2001). No estudo de Vieira e Palma (2005), os autores dividem as comunidades de pequenos mamíferos em três conjuntos segundo sua composição: comunidades em florestas, comunidades em áreas abertas e comunidades em habitats mésicos savânicos. Nesse estudo observamos que as espécies de pequenos mamíferos do campo rupestre, composto por várias espécies do cerrado de ampla distribuição, pode ser dividido em pelo menos dois subgrupos (Figura 4b).

Em resumo, este estudo mostrou que as áreas de campo rupestre do Distrito Federal, apresentam uma composição faunística de pequenos mamíferos similares as áreas de cerrado sensu lato. O número de espécies variou entre os sítios, apresentando uma riqueza comparável as áreas de campo e cerrado sensu stricto. As maiores diferenças observadas entre as comunidades de pequenos mamíferos do campo rupestre no Distrito Federal, estavam associadas com diferenças na posição geográfica dos sítios e na estrutura do habitat. Os resultados sugerem que as comunidades de pequenos mamíferos amostradas pertencem a duas regiões biogeográficas distintas o que pode explicar a diferença faunística encontrada entre a APA de Cafuringa e a APA Gama e Cabeça de Veado.

\section{Agradecimentos}

À Vanessa Teruya, Carla Andrade, Nelma Toledo, Mardônio Timo pelo auxilio no campo. Ao Emerson Vieira e ao Mário Almeida pelas sugestões e auxilio no manuscrito. A Capes e ao CNPq pelo auxilio financeiro na pesquisa.

\section{Referências}

ALHO, C.J.R. 1981. Small mammal populations of Brazilian Cerrado: the dependence of abundance and diversity on habitat complexity. Rev. Bras. Biol. 41(1):223-230.

ALHO, C.J.R., PEREIRA, L.A. \& PAULA, A.C. 1986. Patterns of habitat utilization bysmall mammal populations in cerrado biome of central Brazil. Mammalia. 50(4):447-460.

AUGUST, P.V. 1983 .The role of habitat and heterogeneity in structuring tropical mammal communities. Ecology. 64(6):1495-1507.

BEGON, M., TOWNSEND, C.R. \& HARPER, J.L. 2006. Ecology: from individuals to ecosystems. Blakwell Publishing, Oxford.

BONVICINO, C.R., LEMOS, B. \& WEKSLER, M. 2005. Small mammals of Chapada dos Veadeiros National Park (Cerrado of Central Brazil): ecologic, karyologic, and taxonomic considerations. Braz. J. Biol. 65(3):395-406.

BONVICINO, C.R., LIMA, J.F.S. \& ALMEIDA, F.C. 2003. A new species of Calomys Waterhouse (Rodentia, Sigmodontinae) from the Cerrado of Central Brazil. Rev. Bras.Zool. 20(2):301-307.

BONVICINO, C.R., LINDBERGH, S.M. \& MAROJA, L.S. 2002. Small nonflying mammals from conserved and altered areas of Atlantic Forest and 
Cerrado: comments on their potential use for monitoring environment. Braz. J. Biol. 62(4B):765-774.

BRIANI, D.C., PALMA, A.R.T., VIEIRA, E.M. \& HENRIQUES, R.P.B. 2004. Post-fire succession of small mammals in the Cerrado of central Brazil. Biod. Conserv.13(5):1023-1037.

BROWN, J.H. 1973. Diversity of seed- eating desert rodents in sand dune habitats. Ecology 54(4):775-787.

BROWN Jr., K.S. \& GIFFORD, D.R. 2002. Lepidoptera in the cerrado landscape and the conservation of vegetation, soil and topographical mosaics. In The Cerrados of Brazil: ecology and natural history of a Neotropical Savanna (P.S. Oliveira \& R.J. Marquis, eds). Columbia University Press, New York, p. 201-217.

COLLI, G.R. 2005. As origens e a diversificação da herpetofauna do Cerrado. In Cerrado: ecologia, biodiversidade e conservação. (A. Scariot, J.C. Souza-Silva \& J.M. Felfili, eds.). Ministério do Meio Ambiente, Brasília, p. 247-264.

COLWELL, R.K. 2005. EstimateS: statistical estimation of species richness and shared species from samples. Version 8.0. Department of Ecology and Evolutionary Biology, U-43, University of Connecticut, Storrs, USA: http:// viceroy.eeb.uconn.edu/estimates.htm (último acesso em 12/03/2009)

CONCEIÇÃO, A.A., RAPINI, A., PIRANI, J.R., GIULIETTI, A.M., HARLEY, R.M., SILVA, T.R.S., SANTOS, A.K.A., CORREIA, C., ANDRADE, I.M., COSTA, J.A.S., SOUZA, L.R.S., ANDRADE, M.J.G., FUNCHM, R.R., FREITAS, T.A., FREITAS, A.M.M. \& OLIVEIRA, A.A. 2005. Campos Rupestres. In Biodiversidade e conservação da Chapada Diamantina (F.A. Junca, L. Funch \& W. Rocha, eds.). Ministério do Meio Ambiente, Brasília, p. 153-167.

DIETZ, J.M. 1983. Notes on the natural history of some small mammals in central Brazil. J. Mam. 64(3):521-523.

FONSECA, G.A.B. \& REDFORD, K.H. 1984. The mammals of IBGE's ecological reserve, Brasília, and an analysis of the role of gallery forests in increase diversity. Rev. Bras. Biol. 44(4):517-523.

FORTES, P.T.F.O., OLIVEIRA, G.I.M., CREPANI, E. \& MEDEIROS, J.S. 2007. Geoprocessamento aplicado ao planejamento e gestão ambiental na área de proteção ambiental de Cafuringa, Distrito Federal. In Anais XIII Simpósio Brasileiro de Sensoriamento Remoto. INPE, Florianópolis, p. 2613-2620.

FOX, B.J. \& FOX, M.D. 2000. Factors determining mammal species richness on habitat islands and isolates: habitat diversity, disturbance, species interactions and guild assembly rules. Glo. Ecol. Biog.. 9:19-37.

GIULIETTI, A.M. \& PIRANI, J.R. 1988. Patterns of geographic distribution of some plant species from the Espinhaço range, Minas Gerais and Bahia. In Proceedings of a Workshop of a Neotropical Distribution Patterns (W.R. Heyer \& P.E. Vanzolini, eds.)Academia Brasileira de Ciências, Rio de Janeiro, p. 39-69.

GOTELLI, N.J. \& ENTSMINGER, G.L. 2004. EcoSim: null models software for ecology. Version 7. Acquired Intelligence Inc. \& Kesey-Bear. Jericho, VT 05465 : http://garyentsminger.com/ecosim/index.htm (último acesso em 8/03/2009).

HARLEY, R.M. 1988. Evolution and distribution of Eriope (Labiatae) and its relatives in Brazil. In Proceedings of a Workshop of a Neotropical Distribution Patterns (W.R. Heyer \& P.E. Vanzolini, eds.). Academia Brasileira de Ciências, Rio de Janeiro, p. 71-121.

HENRIQUES, P.B.H. \& ALHO, C.J.R. 1991. Microhabitat selection by two rodent species in the Cerrado of Central Brazil. Mammalia. 54:49-56.

HENRIQUES, R.P.B., BIZERRIL, M.X.A. \& KOHLDSORF, T. 1997. Abundância, riqueza e seleção de habitat de pequenos mamíferos dos cerrados do Brasil Central. In Contribuição ao conhecimento ecológico do Cerrado (L.L. Leite \& C.H. Saito, eds.).Editora UnB, Brasília, p. $127-$ 130. HENRIQUES, R.P.B., BIZERRIL, M.X.A. \& PALMA, A.R.T. 2000. Changes in small mammal population after fire in a patch of unburned Cerrado in Central Brazil. Mammalia. 64:173-185.

JORGE, M.C.L., PIVELLO, V.R., MEIRELLES, S.T. \& VIVO, M. 2001. Riqueza e abundância de pequenos mamíferos em ambientes de Cerrado e Floresta, na Reserva Cerrado do Pé-de-gigante, Parque Estadual de Vassununga (Santa Rita do Passa Quatro, SP). Naturalia. 26:287-302.

KERLEY, G.I.H. 1992. Ecological correlates of small mammal community structure in the semi-arid Karoo, South Africa. J. Zool. 227:17-27.

LACHER Jr, T.E. \& ALHO, C.J.R. 1989. Microhabitat use among small mammals in the Brazilian Pantanal. J. Mam. 70(2):396-401.

LACHER Jr., T.E. \& ALHO, C.J.R. 2001. Terrestrial small mammal richness and habitat associations in an Amazon Forest-Cerrado Contact Zone. Biotropica. 33(1):171-181.

MACARTHUR, R.H., RECHER, H. \& CODY, M.L. 1966. On the relation between Habitat selection and species diversity. Am. Nat. 100(913):319-332.
MAC ARTHUR, R.H. \& MAC ARTHUR, J.W. 1961. On bird species diversity. Ecology. 42:594-598.

MAGURRAN, A.E. 2003. Measuring biological diversity. Blackwell Publishing, Oxford.

MARES, M.A. 1992. Neotropical Mammals and the Myth of Amazonian Biodiversity. Science. 225:976-979.

MARES, M.A. \& ERNEST, K.A. 1995. Population and community ecology of small mammals in a gallery forest of central Brazil. J. Mam. 76(3):750-768.

MARES, M.A., ERNEST, K.A. \& GETTINGER, D.D. 1986. Small mammal community structure and composition in the cerrado province of central Brazil. J. Trop. Ecol. 2:289-300.

MARINHO FILHO, J., REIS, M.L., OLIVEIRA, P.S., VIEIRA, E.M. \& PAES, M.N. 1994. Diversity standards and small mammal numbers: conservation of the cerrado biodiversity forests in the zoogeography of the cerrado's nonvolant mammalian fauna. An. Acad. Bras. Ci., 66(supl.1):149-156.

MARINHO FILHO, J., RODRIGUES, F.H.G. \& GUIMARÃES, M.M. 1998. A fauna de vertebrados da estação ecológica de águas emendadas. Secretaria de Meio Ambiente do Distrito Federal, IEMA, Brasília.

MARINHO FILHO, J., RODRIGUES, F.H.G. \& JUAREZ, K.M. 2002. The Cerrado Mammals: diversity, ecology and natural history. In The Cerrado of Brazil (P.S. Oliveira \& R.J. Marquis, eds.). Editora Columbia University, Nova York, p. 267-284

MCCUNE, B. \& MEFFORD, M.J. 1999. Multivariate analysis of ecological data. Version 4.20. Mjm Software, Oregon.

MÜLLER-DOMBOIS, D. \& ELLENBERG, H. 1974. Aims and methods in vegetation ecology. John Wiley \& Sons, New York.

OLIVEIRA, J.A. \& PESSÔA, L.M. 2005. Mamíferos da Chapada Diamantina, Bahia. In Biodiversidade e conservação da Chapada Diamantina (F.A. Juncá, L. Funch \& W. Rocha, eds.). Ministério do Meio Ambiente, Brasília. p. 377-405.

RATTER, J.A., BRIDGEWATER, S. \& RIBEIRO, J.F. 2003. Analysis of the floristic composition of the Brazilian cerrado vegetation III: comparation of the woody vegetation of 376 areas. Edinburgh. J. Bot. 60(1):57-109.

REDFORD, K.H. \& FONSECA, G.A.B. 1986. The role of gallery forests in the zoogeography of cerrado's non-volant mammalian fauna. Biotropica. 18:126-135.

RIBEIRO, R. \& MARINHO FILHO, J. 2005. Estrutura de Comunidades de Pequenos Mamíferos na Estação Ecológica de Águas Emendadas, Planaltina, Distrito Federal. Rev. Bras. Zool. 22(4):898-907.

ROSENZWEIG, M.L. \& WINAKUR, J. 1969. Population ecology of desert rodent communities: habitats and environmental complexity. Ecology. 50:558-572.

SANDERS, H.L. 1968. Marine benthic diversity: a comparative study. Am. Nat. 102:243-282.

SNEATH, P.H.A. \& SOKAL, L.R.R. 1973. Numerical taxonomy. W. H. Freeman, San Francisco.

VIEIRA, E.M. 1999. Small mammal communities and fire in the Brazilian Cerrado. J. Zool. 249:75-80.

VIEIRA, E.M. \& MARINHO FILHO, J. 1998. Pre and post-fire habitat utilization by rodents of cerrado from Central Brazil. Biotropica. 30(3):491-496.

VIEIRA, E.M. \& PALMA, A.R.T. 2005. Pequenos mamíferos de Cerrado: distribuição dos gêneros e estrutura das comunidades nos diferentes habitats. In Cerrado: ecologia, biodiversidade e conservação (A. Scariot, J.M. Felfili e J.C. Sousa-Silva, eds.).Ministério do Meio Ambiente, Brasília, p. 265-282.

WEKSLER, M. \& BONVICINO, C.R. 2005. Taxonomy of pigmy rice rats genusOligoryzomys bangs, 1900 (Rodentia, Sigmodontinae) of the Brazilian Cerrado, with the description of two new species. Arq. Mus. Nac. 63(1):113-130.

WILLIAMS, S.E., MARSH, H. \& WINTER J. 2002. Spatial scale, species diversity, and habitat structure: small mammals in australian tropical rain forest. Ecology. 83(5): 1317-1329.

ZAR, J.H. 1999. Biostatistical analysis. 4 ed. Prentice-Hall, New Jersey. 